\title{
Hematoma subdural espinal no traumático
}

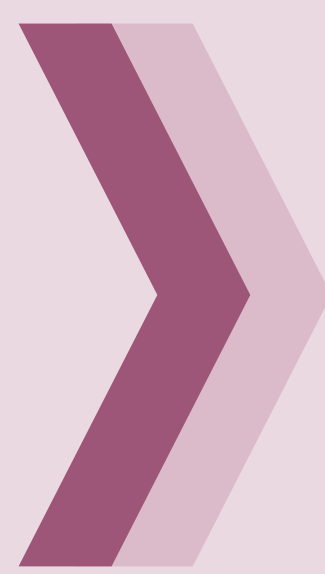

\author{
Nontraumatic spinal \\ subdural hematoma
}

\section{Carlos Casallo-Quiliano, ${ }^{1}$ Dennis Dávila-García, ${ }^{2}$ Catherine Ruiz-Perea, Ronald Pineda-García ${ }^{1}$}

1. Servicio de Neurocirugía, Hospital Militar Central, Lima, Perú.

2. Servicio de Neurología, Hospital Militar Central, Lima, Perú.

\section{RESUMEN}

Un paciente varón de 88 años, en diálisis por insuficiencia renal crónica, presentó cervicalgia intensa $y$, progresivamente, déficit sensitivo motor completo dependiente de ventilación mecánica. La tomografía cervicodorsal y la resonancia magnética (RM) inicial sugirieron varios diagnósticos, entre ellos hematoma subdural espinal. El tiempo de protrombina y el INR estaban ligeramente fuera del rango normal y las plaquetas eran normales. La RM contrastada hecha al cuarto día indicaba la probable presencia de un hematoma espinal. Se realizó una punción lumbar, la que confirmó in hematoma subdural espinal en fase crónica. Se evacuó $60 \mathrm{~mL}$, sin obtener mejora clínica neurológica. No fue posible realizar una RM de control, debido a la condición clínica del paciente. El paciente falleció debido a una sepsis de foco respiratorio.

Palabras claves: hematoma subdural espinal espontáneo; coagulopatía; punción lumbar.

\begin{abstract}
A 88-year-old male receiving hemodialysis for chronic renal insufficiency, developed cervical pain and progressively motor/sensitive deficit with mechanic ventilation support. Computed tomography and Magnetic resonance imaging (MRI) admission showed a variety of diagnostics, inclunding spinal subdural hematoma. Prothrombin time and international normalized ratio (INR) were slightly out of range, with normal platelets amount. MRI after four days found a spinal subdural hematoma, confirmed with lumbar puncture. Sixty cc was evacuated without neurologic amelioration. A new MRI was impossible to perform. He died due a respiratory sepsis.
\end{abstract}

KEY WORDS: spinal subdural hematoma; coagulopathy; lumbar punction. 


\section{INTRODUCCIÓN}

El hematoma subdural espinal (HSDE) es una condición médica muy rara y de riesgo. La formación de un HSDE agudo puede ser debido a causas iatrogénicas como una punción lumbar o una anestesia epidural, muchas veces asociado con anormalidades de la coagulación., ${ }^{1,2}$ Sin embargo, la aparición espontánea debe ser además considerada en pacientes con tratamiento anticoagulante 0 en aquellos con trastornos hematológicos, quienes presentan signos rápidos de compresión medular espinal o cauda equina. Schiller et al. ${ }^{2}$ reportaron el primer caso de HSDE en 1948. En una revisión hecha por Domenicucci et al. ${ }^{3}$, se presentron 21 casos de HSDE agudo en pacientes con alteraciones de coagulación. Muchos son casos únicos, y los hallazgos de resonancia magnética nuclear (RMN) ayudaron en pocos casos. En este reporte, se presenta un paciente sometido a diálisis por insuficiencia renal crónica que tuvo un HSDE tratado médicamente.

\section{CASO CLÍNICO}

Paciente varón de 88 años, con antecedente de hipertensión arterial, insuficiencia cardiaca congestiva e insuficiencia renal crónica (IRC), que, inmediatamente después de una hemodiálisis de rutina, presentó dolor cervical agudo, que después de tres horas se acompaña de disminución de fuerza de las cuatro extremidades. Por esta razón, es llevado a emergencia.

El examen físico mostró un paciente despierto, con paraplejia flácida, nivel sensitivo D2, sin control de esfínteres. Una hora después presenta cuadriplejia e insuficiencia respiratoria, por lo que es intubado y hospitalizado en cuidados intensivos, con ventilación mecánica.

Las tomografías espiral multicorte (TEM) cerebral y cervicodorsal no fueron concluyentes. La punción lumbar fue hemática.

Los exámenes hematológicos mostraron las plaquetas en $144000 / \mu \mathrm{L}$, un tiempo de protrombina
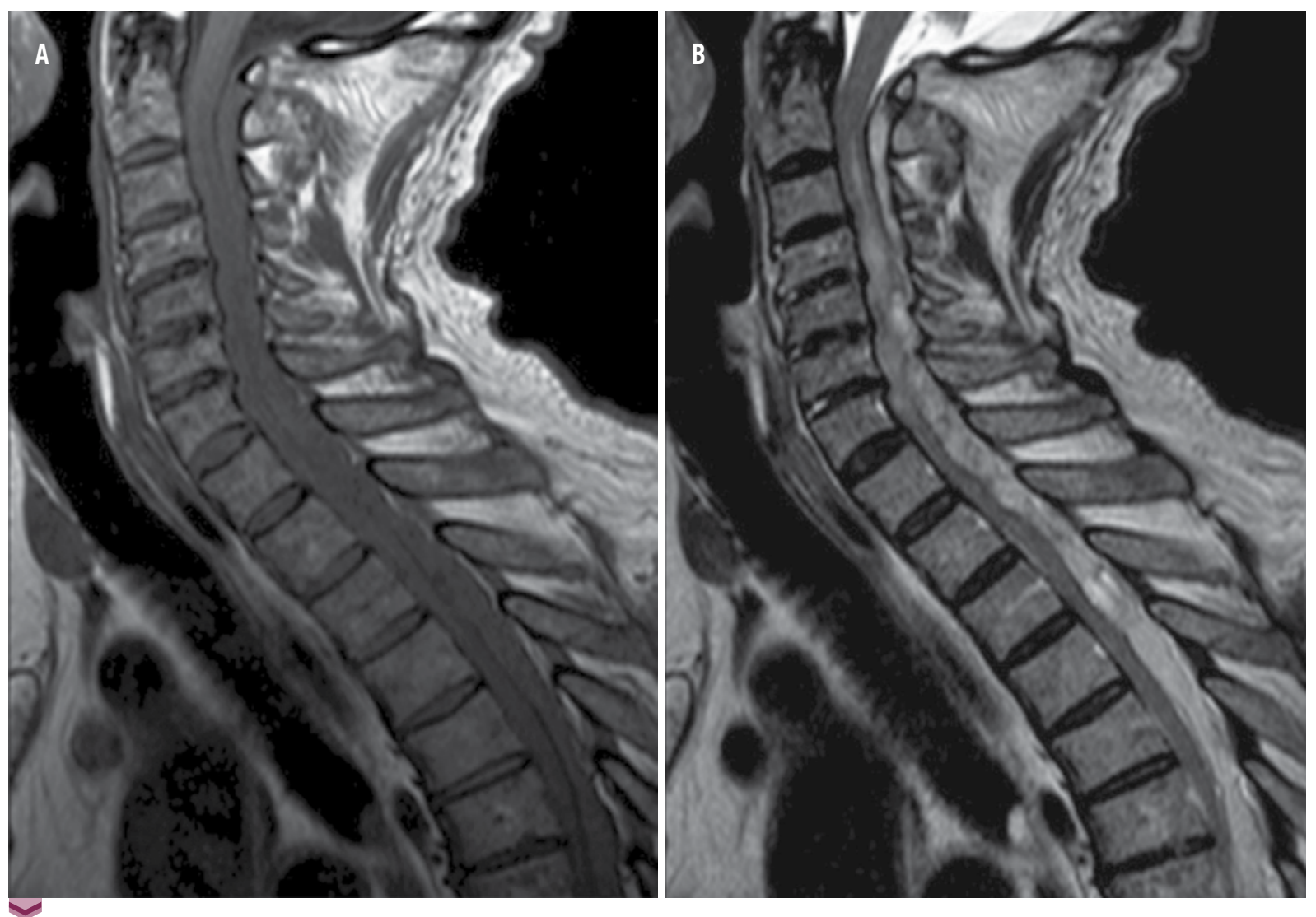

Figura 1. Resonancia magnética inicial. A) Sagital en T1: imagen isointensa en canal espinal. B) En T2: imagen es mixta iso/hiperintensa. 
de 17,8 " a $47,5 \%$ (rango de $70 \%$ a $100 \%$ ), un INR en 1,63 (rango de 0,9 a 1,2). La RMN realizada 24 horas después muestra una masa subdural que se extendía desde C1 hasta L5 (Figura 1), con signos de malacia en médula cervical, sugerente de un proceso expansivo neoformativo versus hematoma subdural subagudo. Luego de cuatro días, se realizó una RMN contrastada cuyas imágenes alejaron la posibilidad de tumor y quedaron como posibilidades diagnósticas hematoma subdural en fase subaguda/crónica y angiolipoma (Figura 2). Entonces, fue sometido a una nueva punción lumbar entre L3 y L4, con aguja N. ${ }^{\circ} 14$. Se obtuvo sangre en estadio subagudo/crónico, lo que confirmó la presencia de un hematoma subdural espinal, y se procedió a evacuar más o menos $60 \mathrm{~mL}$ (Figura 3).

El paciente no mostró mejoría, persistió con el cuadro neurológico instalado y se mantuvo en ventilación mecánica. Por las condiciones del paciente, no se pudo realizar otra RMN de control. Fallece al día 14 como producto de una sepsis de foco respiratorio.

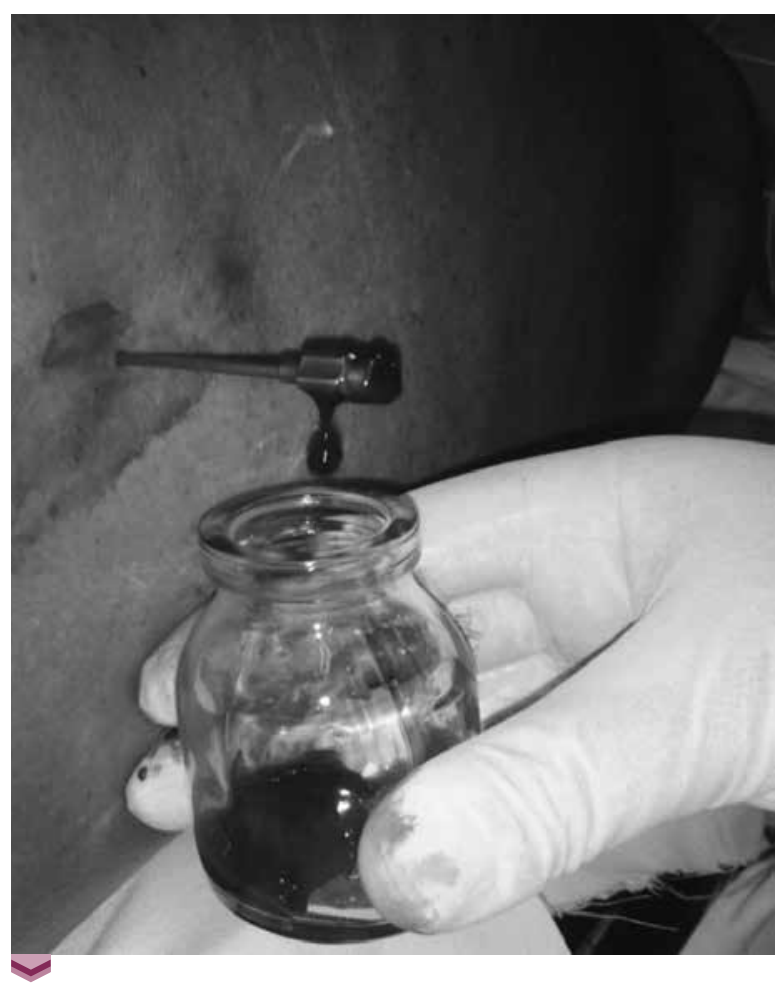

Figura 3. Punción lumbar al cuarto día, se obtuvo $60 \mathrm{~mL}$ de sangre en fase subaguda/crónica.
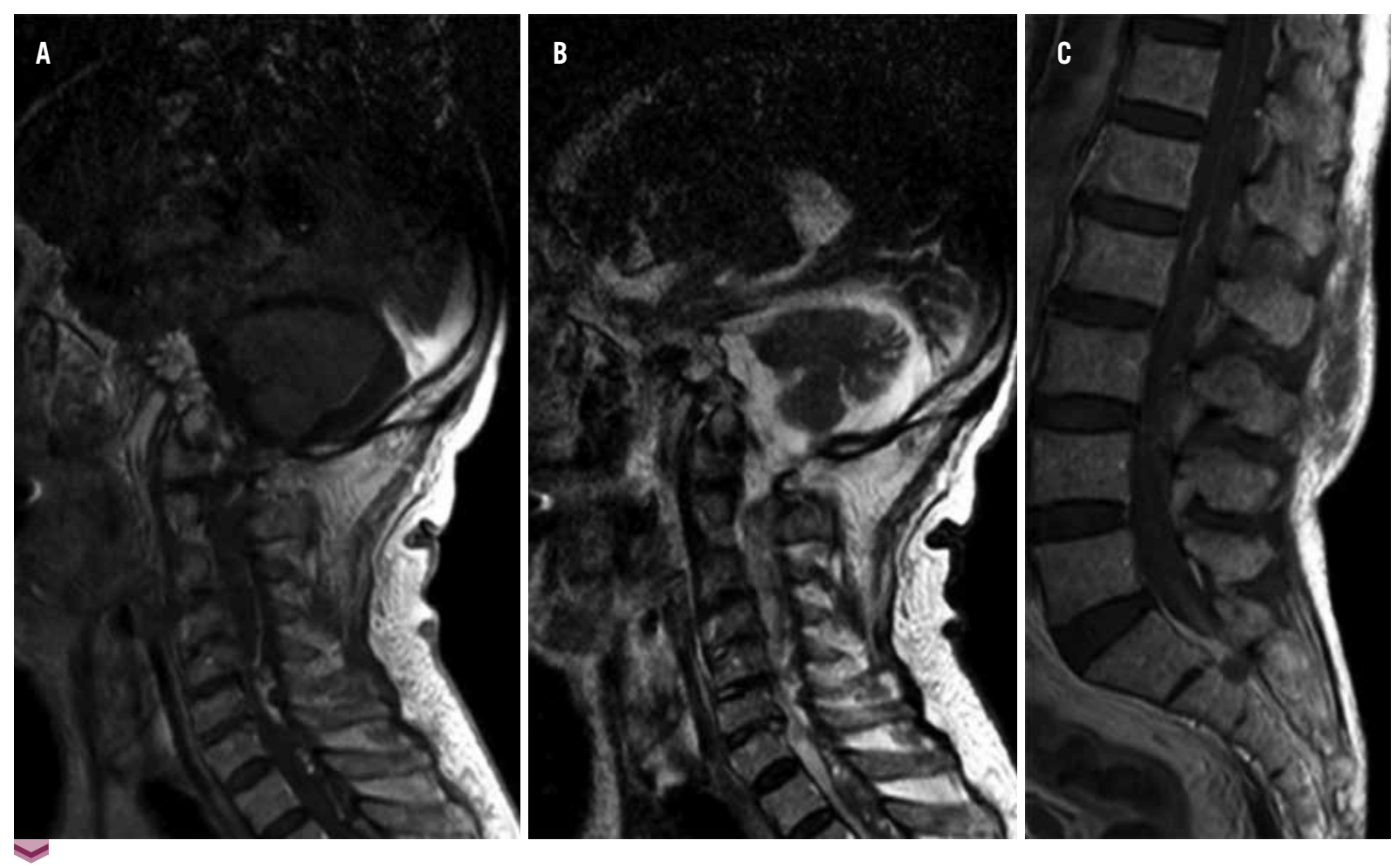

Figura 2. Resonancia magnética contrastada realizada cuatro días despué. A) T1 muestra imagen isointensa.B) T2 con imagen mixta iso/hiperintensa. C) T1 con imagen isointensa en región lumbosacra. 


\section{DISCUSIÓN}

Existen pocos reportes de pacientes con hematomas subdurales espinales no traumáticos. Las causas de HSDE agudos han sido discutidos en la literatura internacional, aunque existen controversias en cuanto al origen de sangrado y al mecanismo de formación del hematoma. Muchos autores han postulado que el incremento súbito de la presión en la cavidad torácica o abdominal podría aumentar la presión en los vasos espinales, particularmente las venas radiculomedulares, porque ellas cruzan el espacio subdural y subaracnoideo., ${ }^{1,2,4}$ Si la presión del líquido cefalorraquídeo (LCR) no puede neutralizar inmediatamente esta fuerza, podría existir ruptura de vasos. Sin embargo, el espacio subdural espinal no contiene vasos sanguíneos grandes, solo una delicada red de vasos en los márgenes laterales de la duramadre. ${ }^{4,5}$

Haines et al. ${ }^{6}$ describen que la duramadre está compuesta externamente por fibroblastos elongados y aplanados y por abundantes cantidades de colágeno extracelular. En la unión duramadre-aracnoides, describe una capa celular dural caracterizada por fibroblastos aplanados, sin colágeno extracelular, espacios extracelulares y pocas células de unión. Esta capa interna de la duramadre es estructuralmente débil. De acuerdo a este autor, no existe un espacio natural en la unión duramadre-aracnoides, entonces los llamados hematomas subdurales podrían ser considerados como hematomas del borde dural. Estos hallazgos también han sido sugeridos por estudios patológicos y clínicos. ${ }^{7,8}$

En el caso presentado, la hemorragia habría sido causada por la ruptura de un pequeño vaso extraaracnoideo localizado en la cara interna de la duramadre. Esta hemorragia se extendió ampliamente en el canal espinal a través de la capa celular del borde dural.

Todos los pacientes reportados en la literatura presentaron dolor espinal como principal síntoma, seguido rápidamente por dolor radicular. En pocas horas, se instaló el déficit motor y sensitivo y, además, pérdida de control de esfínteres. El desarrollo de paraplejia tomó entre 10 y 26 horas. El examen clínico no ayudó a identificar la naturaleza de la lesión, sin embargo, el factor más importante que si favorecía al desarrollo del hematoma fue el antecedente de anticoagulación, a pesar que los valores de INR y el tiempo de protrombina (TP) estaban ligeramente por encima del rango terapéutico deseado.

El paciente presentó dolor cervical intenso y tres horas después trastornos motor y sensitivo, que progresivamente se agravaron. Fueron necesarias la intubación y la ventilación mecánica a las siete horas de iniciado el cuadro. El antecedente de IRC con hemodiálisis rutinaria, tres veces a la semana, y los valores de INR y de TP ligeramente elevados con relación a los valores normales hicieron pensar en la presencia de un hematoma.

Los reportes indican que el método de elección para diagnosticar un HSDE es la RMN.1,7,-12 En imágenes sagitales, un HSDE aparece como aglutinado y de forma cóncava, contrariamente los hematomas epidurales espinales (HES) tienen forma convexa. Post et al. ${ }^{10}$ muestran que la tomografía (TC) es de mejor ayuda en distinguir un HSDE de un HES. Clásicamente, en T1-w, las imágenes tienen hipo 0 isointensidad, y en T2-w son muy hipointensas, debido a la presencia de deoxihemoglobina.

En este caso, el paciente fue sometido inicialmente a una TC de columna cervicodorsal, que no fue concluyente. La RMN mostró en T1-w una imagen hipointensa y en T2-w, una isointensa, que, ante la poca frecuencia de esta patología, sugirió varias probabilidades diagnósticas. La segunda RMN y la punción lumbar evacuatoria fueron los exámenes con los que se diagnosticó el HSDE.

Muchos autores postulan que un HSDE agudo es una emergencia quirúrgica, con buen pronóstico en pacientes que son diagnosticados y operados rápidamente, cualquiera fuese el estado neurológico. 1-3,9 Cuando revisaron la literatura, Domenicucci et al. ${ }^{3}$ encontraron que la localización anatómica influye en el pronóstico. Si está localizado en el espacio subdural o si además produce una hemorragia subaracnoidea, siendo esta última condición de peor pronóstico, en adición al efecto compresivo propio del hematoma. La presencia de sangre en el LCR puede producir reacción fibroproliferativa de la leptomeninge, lo que resulta en fibrosis aracnoidea. ${ }^{13-15}$ 
Otros autores han sugerido que el HSDE localizado a nivel lumbar podría ser manejado exitosamente con drenaje percutáneo. ${ }^{16,17}$ Sin embargo, el riesgo de injuria iatrogénica de vasos sanguíneos es alto y la remoción de coágulos organizados es imposible. Raros casos de recuperación espontánea han sido reportados, ${ }^{18,19}$ lo que sugiere que el tratamiento conservador puede ser empleado en casos selectos.

En el paciente presentado, cuatro días después, se realizó una segunda punción lumbar diagnóstica, que mostró sangre en estadio subagudo/ crónico, por lo que se procedió a la evacuación de aproximadamente $60 \mathrm{~mL}$, sin obtener mejora neurológica. No se pudo realizar una nueva RMN de control, por el estado neurológico y la dependencia de ventilación mecánica. Posteriormente, el paciente fallece por una sepsis de foco respiratorio.

\section{CONCLUSIÓN}

El hematoma intraespinal es la primera presunción diagnóstica en pacientes con trastornos de coagulación primarios 0 secundarios que presentan signos espontáneos de compresión medular o de cauda equina. Es importante saber reconocer esta condición como una emergencia quirúrgica, y hospitalizar al paciente en una unidad especializada. La descompresión quirúrgica realizada muchas horas después de la aparición de un déficit sensitivo y motor completo aumenta el riesgo de tener un pobre pronóstico funcional. Las imágenes de resonancia magnética sagitales en T1-w y T2-w ayudan al diagnóstico temprano y a una evacuación quirúrgica oportuna. Un seguimiento por resonancia podría mostrar atrofia medular y fibrosis aracnoidea que pueden explicar parcialmente el mal pronóstico funcional.

\section{REFERENCIAS BIBLIOGRÁFICAS}

I. Levy JM. Spontaneous lumbar subdural hematoma. Am J Neuroradiol. 1990; I 1:780-781.

2. Schiller F, et al. Surgery in hemophilia:A case of spinal hematoma producing paraplegia. Lancet. 1948;2:842-845.

3. Domenicucci M, Ramieri A, Ciappetta P, Delfini R. Nontraumatic acute spinal subdural hematoma: report of five cases and review of the literature.J Neurosurg. 1999;9I(I Suppl):65-73.

4. Gillilan LA.Veins of the spinal cord. Anatomic details; suggested clinical applications. Neurology. $1970 ; 20(9): 860-868$

5. Grunberg A, et al. Hematomes sous-duraux rachidiens: Presentation de deux cas explores en IRM. J Radiol. 1993;74:29I-295.

6. Haines DE, Harkey HL, al-Mefty O.The "subdural" space: A new look at an outdated concept. Neurosurgery. 1993;32: I I I-I 20.

7. Russel NA, Benoit BG. Spinal subdural hematoma:A review. Surg Neurol. 1983;20:133-137.

8. Schwartz FT, Sartawi MA, Fox JL. Unusual hematomas outside the spinal cord: Report of two cases. J Neurosurg. 1973;39:249251.

9. Johnston RA.The management of acute spinal cord compression. J Neurol Neurosurg Psychiatry. 1993;56: 1046- 1054.

10. Post MJD, Becerra JL, Madsen PW, PuckettW, Quencer RM, Bunge $\mathrm{RP}$, et al. Acute spinal subdural hematoma: $M R$ and $C T$ findings with pathologic correlates. Am J Neuroradiol. 1994;15:18951905.

I I. Quiles M, Marchisello PJ,Tsairis P. Lumbar adhesive arachnoiditis: Etiologic and pathologic aspects. Spine. 1978;3:45-50.
12. Kulkarni AV,Willinsky RA, Gray T, Cusimano MD. Serial magnetic resonance imaging findings for a spontaneously resolving spinal subdural hematoma: Case report. Neurosurgery. 1998;42:398$40 \mathrm{I}$.

13. Langmayr JJ, Ortler M, Dessl A, Twerdy K, Aichner F, Felber S. Management of spontaneous extramedullary spinal hematomas: Results in eight patients after MRI diagnosis and surgical decompression.J Neurol Neurosurg Psychiatry. 1995;59:442-447.

14. Sakata T, Kurihara A. Spontaneous spinal subdural hematoma: A case report. Spine. 1984;9:324-326.

15. Kok AJ, Verhagen WI, Bartels RH, van Dijk R, Prick MJ. Spinal arachnoiditis following subarachnoid haemorrhage: Report of two cases and review of the literature. Acta Neurochir. (Wien) 2000; 142:795-799.

16. Mavroudakis N, Levivier M, Rodesch G. Central cord síndrome due to a spontaneously regressive spinal subdural hematoma. Neurology. 1990;40: 1306-1308.

17. Morandi X, Carsin-Nicol B, Brassier G, Scarabin JM. MR demostration of spontaneous acute spinal subdural hematoma.J Neuroradiol. 1998;25:46-48.

18. Lee Jl, Hong SC, Shin HJ, Eoh W, Byun HS, Kim JH. Traumatic spinal subdural hematoma: Rapid resolution after repeated lumbar spinal puncture and drainage. J Trauma. 1996;40:654-655.

19. Morandi X, Riffaud L, Chabert E, Brassier G.Acute Non traumatic spinal subdural hematomas in three patients. Spine. 200।;26:547551 .

\section{Correspondencia}

Dr. Carlos Casallo-Quiliano: adriancasallo@yahoo.es

Fecha de recepción: 3 de octubre de 2014

Fecha de aceptación: 10 de octubre de 2014

\section{Conflictos de interés}

El autor declara no tener conflictos de interés durante el planteamiento, ejecución de la investigación y la elaboración del artículo para su publicación. 